\title{
LES and DNS of the Flow with Heat Transfer in Rotating Cavity
}

\author{
Ewa Tuliszka-Sznitko, Wojciech Majchrowski \\ Institute of Thermal Engineering, Poznań University of Technology \\ 60-965 Poznań, Poland \\ e-mail: ewa.tuliszka-sznitko@put.poznan.pl
}

(Received: 1 December 2009; revised: 2 March 2010; accepted 10 March 2010; published online: 20 April 2010)

\begin{abstract}
In the present paper we summarized our numerical investigations on the flow with heat transfer in rotating cavity performed by DNS (Direct Numerical Simulation) and LES (Large Eddy Simulation). We considered different geometrical configurations and different flow and thermal conditions. All presented computations have been performed in Poznań Supercomputing and Networking Center. The objective of our investigations was to analyze the coherent structures of transitional and turbulent flows and to compute statistical parameters, i.e. turbulent heat fluxes, the Reynolds stress tensor components, the turbulent Prandtl number and others. In the LES we used a version of the dynamic Smagorinsky eddy viscosity model proposed by Meneveau et al. (A Lagrangian dynamic subgrid-scale model of turbulence, J. Fluid Mech., vol. 319, 1996), in which the Smagorinsky coefficient at a given position $x$ depends on the history of the flow along the fluid particle pathline.
\end{abstract}

Key words: laminar-turbulent transition, flow instability, rotating cavity, spectral methods, DNS, LES

\section{INTRODUCTION}

This paper is a review of our study on the numerical prediction of the transitional and turbulent flow with heat transfer in rotating cavities. The configurations with throughflow are also considered. The main motivation of our work was to analyze the laminar-turbulent transition process as well as to analyze properties of the turbulence of the non-isothermal flow dominated by Coriolis and centrifugal forces. Additionally, the flow between rotating disks is one of the simplest 3D flows highly suitable for investigating the effect of mean flow parameters on the turbulence and transitional structures. The problem is very interesting not only from the fundamental point of view, but it is also a topic of practical importance. The flow in the rotating cavity is of great interest for the optimization of turbomachinery air-cooling devices. The experimental investigations in the rotating cavities are very difficult and expensive. In this situation numerical simulations, particularly DNS (Direct Numerical Simulation) and LES (Large Eddy Simulation), are valuable tools which can deliver precise knowledge on the flow structure and temperature distributions in the rotating cavity.

One of the first experimental investigations of the flow in rotor/stator configurations was performed by Daily and Nece [1]. Daily and Nece [1] found that the flow structure depends on the Reynolds number $R e_{1}=\Omega_{1} R_{1}^{2} / v$ and aspect ratio $L=\left(R_{1}-R_{0}\right) / 2 h$ (where $R_{0}$ and $R_{1}$ are the radiuses of the inner and outer cylinder, respectively, $h$ is half of the interdisks spacing, $\Omega_{1}$ is the angular rotation of the upper disk, $v$ is the kinetic viscosity). Heat transfer at a single rotating disk was investigated by McComas and Hartnett [2], Popiel and Bogusławski [3], Littell and Eaton [4], Elkins and Eaton [5]. Nikitenko [6] performed experimental investigation of the flow in the enclosed rotor/stator cavity (where both disks were isothermal) for a wide range of aspect ratio $11.8<L<55.5$ and for $R e<10^{6}$. Owen and Pincombe [7] investigated experimentally the flow in the cavity with throughflow: cooling air was introduced through the hole in the centre of an adiabatic stator, and parabolic profile of temperature was kept on rotor. Schukin and Olimpiev [8] measured the average Nusselt numbers in the rotating cavity of aspect ratio $L=15.5$. All earlier theoretical and numerical works on the flow with heat transfer in rotating disks systems were summarized by Owen and Rogers $[9,10]$. Mochizuki et al. [11] performed measurements to determine fluid flow and heat transfer in the co-rotating rotor/rotor cavity of aspect ratio $L=78.5$ with throughflow (they presented the distributions of the local Nusselt number along the disks). Itoh et al. [12] performed measurements of the mean velocity profiles and the Reynolds stress tensor compo- 
nents distributions in the enclosed cavity of aspect ratio 12.5 for a wide range of parameters. Djaoui and Debuchy [13] measured the Reynolds stresses in the rotor/stator cavity of large aspect ratio with throughflow using a hot and cold wire anemometry technique. Pellé and Harmand [14] performed measurements over the rotor (in rotor/stator configuration), using a technique based on infrared thermography.

Numerical modeling of the flow in the rotor/stator cavity turned out to be a difficult problem mostly due to the fact that in the cavity areas of laminar, transitional and turbulent flow exist simultaneously, and these areas are completely different in terms of flow properties. The first numerical modeling in the rotor/stator cavity was performed in the 1980s by Morse [15] who used a modified version of the low Reynolds number $k-\varepsilon$ model proposed by Launder and Sharma [16]. Chew and Vaughan [17] solved the Reynolds equations in the rotor/stator cavity using a finite difference method and a mixing length model. Many of the direct simulations (DNS) were dedicated to instability in shrouded rotating cavities: Serre et al. [18, 19], Moisy et al. [20], Jacques et al. [21], Randriamampianina and Poncet [22], Tuliszka-Sznitko et al. [23-25]. The main contribution concerning the turbulent flow (using DNS) was done by Lygren and Andersson [26] who provided a detailed description of the coherent structures in the open rotating cavity. The first LES computations of the flow around a single rotating disk were performed by $\mathrm{Wu}$ and Squires [27]. Then, Andersson and Lygren [28] performed LES numerical computations for enclosed cavity. Séverac et al. [29] used the Spectral Vanishing Viscosity (SVV) method and compared the results with their LDV experimental data. The nonisothermal flow conditions were also considered in some investigations (Tuliszka-Sznitko et al. [24], Randriamampianina et al. [30]) which showed that the thermal effects and the rotation-induced buoyancy influence the stability characteristics and the critical conditions. Tuliszka-Sznitko et al. [24] performed the LES of the non-isothermal flow in the rotor/stator cavity with heated stator and outer cylinder, delivering distributions of the local Nusselt numbers along the stator and rotor for different configurations and Reynolds numbers.

In the present paper we shortly summarized the results obtained by DNS and LES for different configurations and for different flow and thermal parameters. The objective of our investigations was to analyze the structures which appear in disks boundary layers, and to compute statistical parameters. This data can be useful for heat transfer modeling and can help to understand modifications in the turbulence structures introduced by the mean flow skewing.
In Chapters II and III the geometrical and numerical models are analyzed respectively. Our LES investigations were based on a version of the dynamic Smagorinsky eddy viscosity model proposed by Meneveau et al. [31], in which the averaging is performed over the fluid particle pathline. In Sections IV, V and VI we shortly analyzed results obtained for rotor/stator cavity, for counter-rotating rotor/rotor cavity and for rotor/rotor cavity withthroughflow. Conclusions and closing remarks are given in Section VII.

\section{GEOMETRICAL MODEL}

The geometrical model is a co- and counter-rotating cavity (Fig. 1). The faster rotating (upper) disk rotates at uniform angular velocity $\Omega_{1}$. The slower rotating disk rotates at angular velocity $\Omega_{2}=s \Omega_{1}$ where $s$ is the rotation speed rate. Positive $s$ means that both disks rotate in the same direction and negative s means that the disks rotate in opposite directions. The outer cylinder of radius $R_{1}$ and the inner cylinder of radius $R_{0}$ can be attached to the slower or to the faster rotating disk. The flow is controlled by the following physical parameters: the Reynolds number based on the external radius of the disks and on the angular velocity of the faster rotating disk $R e_{1}=\Omega_{1} R_{1} / v$ ( $v$ is the kinetic viscosity), the Reynolds number based on the external radius of the disks and on the angular velocity of slower rotating disk $R e_{2}=\Omega_{2} R_{1} / v$, the aspect ratio $L=\left(R_{1}-R_{0}\right) / 2 h$ where $\mathrm{h}$ is half of the distance between disks, the curvature parameter $R m=\left(R_{1}+R_{0}\right) /\left(R_{1}-R_{0}\right)$, the Prandtl number $\operatorname{Pr}=0.71$. The thermal Rossby number is defined as follows: $B=\beta\left(T_{2}-T_{1}\right)$, where $\beta$ is the thermal expansion coefficient and $T_{1}, T_{2}$ are temperatures of two chosen walls. For the rotor/stator configuration with the outer cylinder attached to the stator, $T_{1}$ is the temperature of the upper rotating disk and the inner cylinder, and $T_{2}$

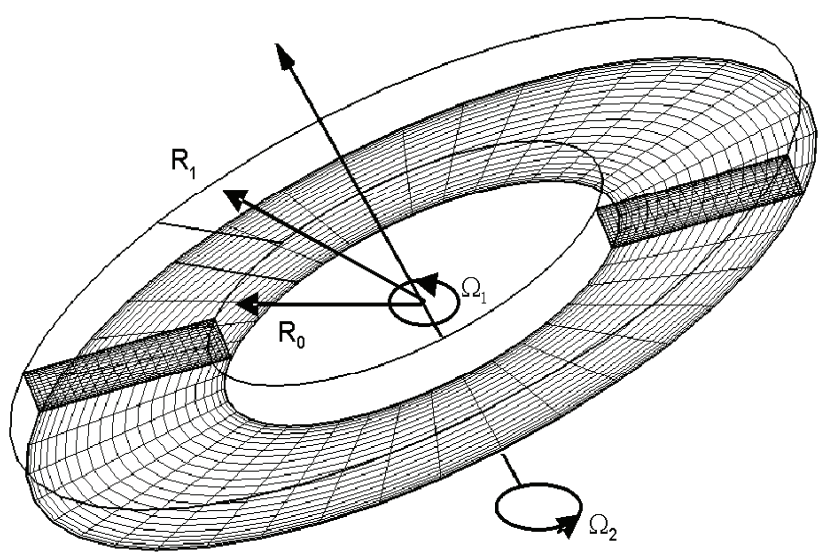

Fig. 1. Schematic picture of the rotating cavity 
indicates temperature of the stator and the outer cylinder. In general, flow can penetrate through the cylinders and through the part of the disks. For the throughflow case the dimensionless flow rate $C w$ is defined as follows: $C w=\dot{V} / \nu R_{1}$ where $\dot{V}$ is a volumetric flow rate.

The flow is described by the continuity, Navier-Stokes and energy equations. The equations are written in a cylindrical coordinate system $(R, \varphi, Z)$ with respect to a rotating frame of reference:

$$
\nabla \cdot \mathbf{V}=0
$$

$$
\begin{aligned}
\rho \frac{\partial \mathbf{V}}{\partial t}+\rho(\mathbf{V} \cdot \nabla) \mathbf{V} & +\rho \Omega \times(\Omega \times R)+2 \rho \Omega \times \mathbf{V}= \\
& =-\nabla P+\mu \Delta \mathbf{V} \\
\frac{\partial T}{\partial t} & +(\mathbf{V} \cdot \nabla) T=a \Delta T
\end{aligned}
$$

where: $t$ is time, $R$ is radius, $P$ is pressure, $\rho$ is density, $\mathbf{V}$ is the velocity vector, $a$ is a thermal diffusivity. To take into account the buoyancy effects induced by the involved body forces, the Boussinesq approximation is used $\rho=\rho\left[1-\beta\left(T_{2}-T_{1}\right)\right]$, where $\beta=-1 / \rho_{r}(\partial \rho / \partial T)_{p}$ is the thermal expansion coefficient, $r$ depicts the reference value. The thermal Rossby number is defined in the following way $B=\beta\left(T_{2}-T_{1}\right)$. The dimensionless temperature is defined in the following manner: $\Theta=\left(T-T_{1}\right) /\left(T_{2}-T_{1}\right)$. The time, length and velocity are normalized as follows: $\Omega_{1}^{-1}, h, \Omega_{1} R_{1}$ (Fig. 1). The dimensionless axial coordinate is $z=Z / h ; z \in[-1,1]$. The radius coordinate is normalized additionally to obtain the domain $[-1,1]$ requested by a spectral method based on the Chebyshev polynomials: $r=\left(2 R /\left(R_{1}-R_{0}\right)-R m\right)$. The dimensionless components of the velocity vector in radial, azimuthal, axial directions and pressure are depicted as follows: $u, v, w$ and $p$. The periodicity condition is used in the azimuthal direction. The no slip boundary condition is applied to all rigid walls, so $u=w=0$. For the azimuthal velocity component the boundary conditions are: $v=0$ on the top faster rotating disk, and $v=(s-1)(R m+r) /(R m+1)$ on the slower rotating disk.

\section{NUMERICAL APPROACH}

The numerical solution is based on a pseudo-spectral collocation Chebyshev-Fourier-Galerkin approximation. The approximation of the flow variables $\Psi=[u, v, w, p . \Theta]^{T}$ is given by a development in truncated series:

$$
\begin{gathered}
\Psi_{N M K}(r, z, \varphi, t)=\sum_{p=-K / 2}^{K / 2-1} \sum_{n=0}^{N} \sum_{m=0}^{M} \hat{\Psi}_{n m p}(t) T_{n}(r) T_{m}(z) e^{i p \varphi} \\
-1 \leq r, z \leq 1 ; \quad 0 \leq \varphi \leq 2 \pi,
\end{gathered}
$$

where $T_{n}(r)$ and $T_{m}(z)$ are Chebyshev polynomials, $t$ is a dimensionless time, $N, K$ and $M$ are the numbers of collocation points in the radial, azimuthal and axial directions. The uniform mesh has been used in azimuthal direction. The spatial resolutions depend on the considered case (the whole number of collocation points which are used in simulation is about $1.8 \times 10^{6}$ ). The time scheme is semi-implicit and second-order accurate. It corresponds to a combination of the second order backward differentiation formula, for the viscous diffusion term, and the AdamsBashforth scheme, for the non-linear terms. The time step is $10^{-4}-10^{-5}$. The method uses a projection scheme to maintain the incompressibility constraint. The numerical algorithm, which we proposed for LES of the nonisothermal flow in the rotating cavity [24, 25], is an extended version of the DNS code for isothermal flow developed by Serre and Pulicani [32]. For the large eddy simulation, after a filtering operation had been applied to the governing equations with a filter width equal to the grid spacing in azimuthal direction, we obtained the filtered equations of motions [24, 25, 33, 34]. Subgrid-scale stress tensor $\sigma_{i j}^{S G S}$ and energy flux $\alpha_{i j}^{S G S}$ are expressed as:

$$
\begin{gathered}
\sigma_{i j}^{S G S}=-2 v_{S G S} \bar{S}_{i j} \\
\alpha_{j}^{S G S}=\frac{v_{S G S}}{\operatorname{Pr}_{S G S}} \frac{\partial \zeta^{k}}{\partial x_{j}} \frac{\partial \Theta}{\partial \zeta^{k}},
\end{gathered}
$$

where

$$
\begin{gathered}
\bar{S}_{i j}=\frac{1}{2}\left(\frac{\partial \zeta^{k}}{\partial x_{j}} \frac{\partial \bar{u}_{i}}{\partial \zeta^{k}}+\frac{\partial \zeta^{k}}{\partial x_{i}} \frac{\partial \bar{u}_{k}}{\partial \zeta^{k}}\right) \\
v_{S G S}=C_{S}^{2} \Delta^{2} \sqrt{2 \bar{S}_{i j} \bar{S}_{i j}}
\end{gathered}
$$

where filtered dependent variables are indicated by overbars, $\Delta$ is the filter width. $\operatorname{Pr}_{S G S}$ is the Prandtl subgrid coefficient. In the above equations $\left(x_{1}, x_{2}, x_{3}\right)=(x, y, z)$ and $\left(\zeta^{1}, \zeta^{2}, \zeta^{3}\right)=(R, \varphi, Z)$. In our computations we used a version of the dynamic Smagorinsky eddy viscosity model proposed by Meneveau et al. [31]; in this approach the required stabilizing averaging is accumulated over the fluid particle pathlines, instead of averaging over the direction of statistical homogenity. The Smagorinsky coefficient is determined by minimizing of the modeling error over the pathline of the fluid particle. 


$$
C_{S}^{2}=\frac{L_{L M}^{n+1}}{L_{M M}^{n+1}},
$$

where

$$
\begin{aligned}
& L_{L M}^{n+1}(x)=H\left(\varepsilon\left(L_{i j} M_{i j}\right)^{n+1}(x)+(1-\varepsilon) L_{L M}^{n}(x-\bar{u} \Delta t)\right)(6 \mathrm{a}) \\
& L_{M M}^{n+1}(x)=\varepsilon\left(M_{i j} M_{i j}\right)^{n+1}(x)+(1-\varepsilon) L_{M M}^{n}(x-\bar{u} \Delta t) \quad(6 \mathrm{~b})
\end{aligned}
$$

In the above equations $x$ is the position of particle in considered time " $n+1$ " and $(x-\bar{u} \Delta t)$ is the position of particle in the previous time step " $n$ ", $\bar{u}$ is the resolved velocity, $H$ is a ramp function $(H(a)=a$ if $a>0$ and zero otherwise), $\varepsilon$ is the weighting function [31]:

$$
\varepsilon=\frac{\Delta t / \tau^{n}}{1+\Delta t / \tau^{n}}
$$

where: $\Delta t$ is the time increment, $\tau$ is the dimensionless time scale over which the averaging is performed. Meneveau et al. [31] give some possible choices for $\tau$. The time scales were chosen by Meneveau et al. [31] based on the requirement that the model should be purely dissipative, ensuring numerical stability. In our simulations we used the following function for the time scale $\tau$ :

$$
\tau=1.5 \Delta\left(M_{i j} M_{i j}\right)^{-1 / 4},
$$

where

$$
\begin{gathered}
M_{i j}=2 \Delta^{2}\left[\left|\widehat{\bar{S} \mid \bar{S}_{i j}}-4\right| \hat{\bar{S}} \mid \hat{\bar{S}}_{i j}\right] \\
L_{i j}=\widehat{\overline{\bar{u}}_{i} \bar{u}_{j}}-\hat{\bar{u}}_{i} \hat{\bar{u}}_{j} .
\end{gathered}
$$

In the above equations $\overline{(\cdot)}$ represents filtering at scale $\Delta$ and $\widehat{()}$ represents filtering at scale $2 \Delta$. For the nonisothermal flow we adopted approach proposed by Lilly [34] to the Lagrangean model. We introduced the $S G S$ Prandtl number [24]:

$$
\frac{1}{P r_{S G S}}=\frac{L_{P R M M}^{n+1}}{L_{R R M L}^{n+1}},
$$

where

$$
\begin{gathered}
L_{P R M M}^{n+1}(x)=H\left(\varepsilon\left(P_{j} R_{j} M_{i j} M_{i j}\right)^{n+1}\left(x^{*}\right)+\right. \\
\left.+(1-\varepsilon) L_{P R M M}^{n}(x-\bar{u} \Delta t)\right) \\
L_{R R M L}^{n+1}(x)=\varepsilon\left(R_{j} R_{j} M_{i j} L_{i j}\right)^{n+1}(x)+ \\
+(1-\varepsilon) L_{R R M L}^{n}(x-\bar{u} \Delta t),
\end{gathered}
$$

where

$$
\begin{gathered}
R_{j}=2 \bar{\Delta}^{2}\left(4|\hat{\bar{S}}| \frac{\partial \hat{\bar{\Theta}}}{\partial x_{j}}-|\overline{\bar{S}}| \frac{\partial \bar{\Theta}}{\partial x_{j}}\right) \\
P_{j}=\hat{\bar{u}}_{j} \hat{\bar{\Theta}}-\widehat{\bar{u}_{j} \bar{\Theta}}
\end{gathered}
$$

\section{ROTOR/STATOR CASE}

For the rotor/stator configuration computations were performed for the following geometrical and physical parameters: aspect ratio $(L=3.0-9.0)$, curvature parameter $(R m=1.5-5.0)$, the thermal Rossby number $B=0.1-0.3$ and the Reynolds number (up to 300000 ). The considered flows are of the Batchelor type, which means that flows consist of two separated boundary layers and an inviscid core. The positive thermal Rossby number $B>0$ (heated stator) means that the buoyancy driven secondary flow enforces the basic rotation driven flow. Figure 2 shows the exemplary axial profiles of the radial velocity components obtained in the middle section of the cavities $(L=5$, $R m=5.0,3.0,1.8, B=0.1, R e=100000)$. From Fig. 2 we can see that the flow consists of two separated boundary layers and an inviscid core where the radial velocity component is nearly zero. The flow is pumped radially outward along the rotor and recirculates along the stator.

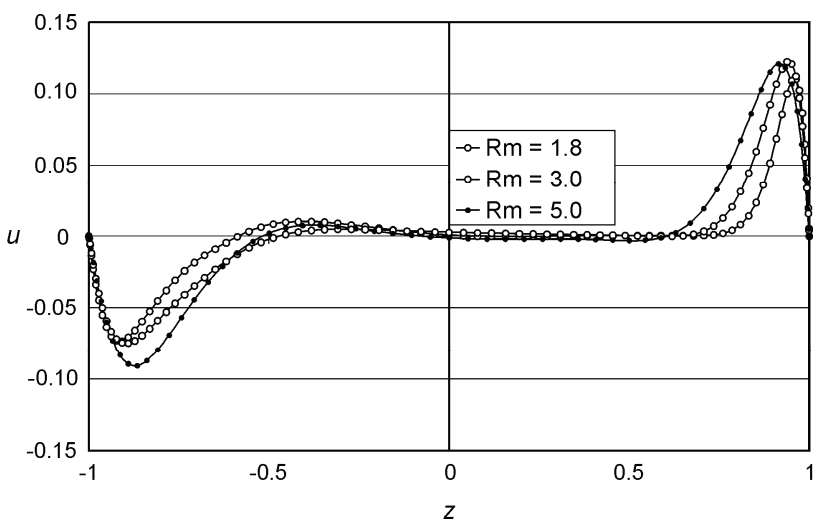

Fig. 2. The axial profiles of the radial velocity component obtained in the middle section of cavities $(L=5, R m=5.0,3.0,1.8$, $B=0.1, R e=100$ 000). Tuliszka-Sznitko et al. [24]

To show the most disturbed regions in the meridian plane, the iso-lines of the axial velocity component obtained for cavities: $(\mathrm{L}=5, R m=1.8, R e=200000),(L=5, R m=3$, $R e=250000),(L=5, R m=5, R e=300000)$ are presented respectively in Fig. $3 \mathrm{a}, \mathrm{b}$ and c. From the figures we can 
a)

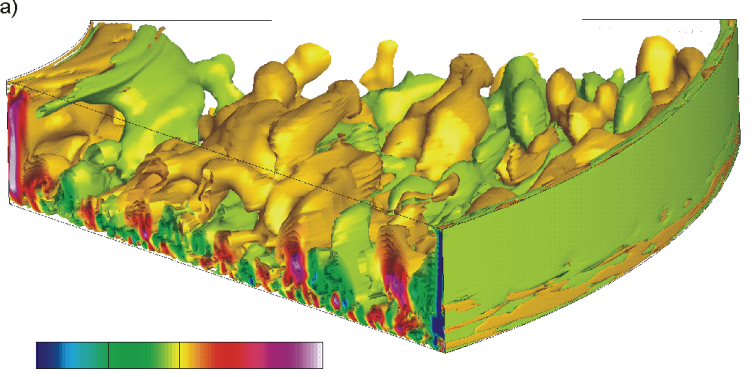

$\begin{array}{lllll}-0.0500 & -0.0250 & 0.0000 & 0.0250 & 0.0500\end{array}$

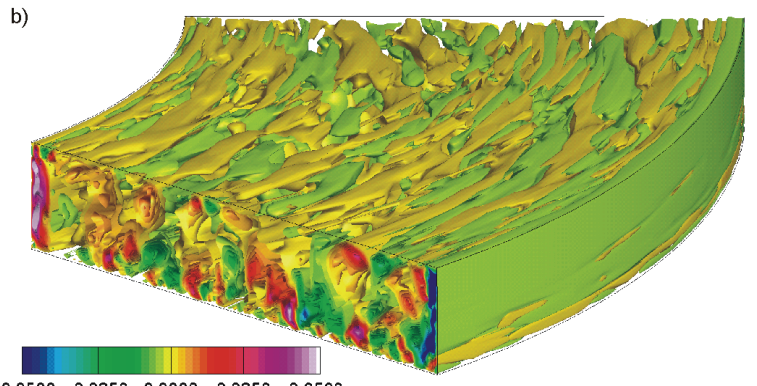

$\begin{array}{lllll}-0.0500 & -0.0250 & 0.0000 & 0.0250 & 0.0500\end{array}$

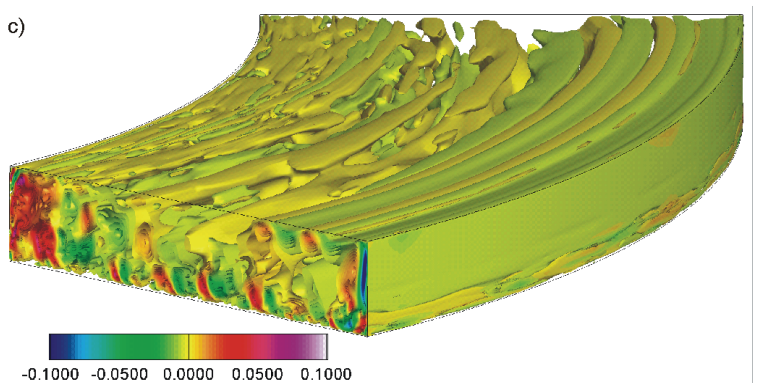

Fig. 3. Isolines of the azimuthal velocity component obtained for: (a) $L=5, R m=1.8, R e=200000$, (b) $L=5, R m=3$, $R e=250000$, c) $L=5, R m=5, R e=300000.0<\varphi<\pi / 4, B=0.1$. Tuliszka-Sznitko and Majchrowski [35]

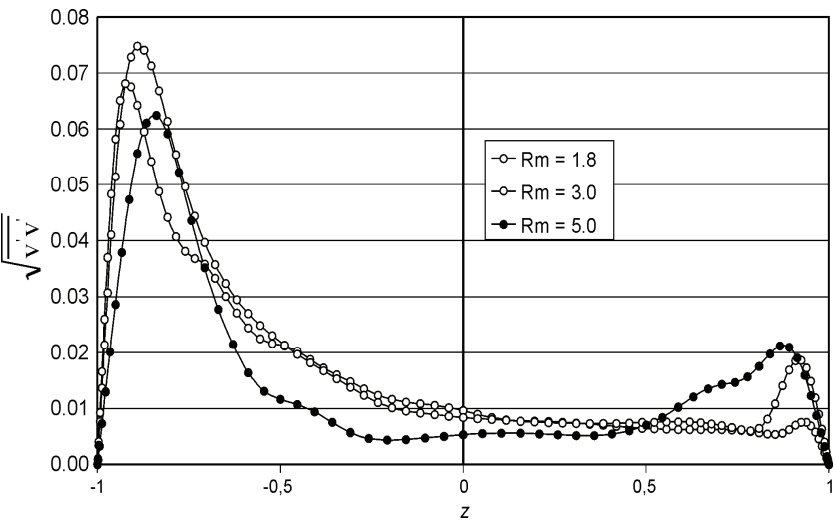

Fig. 4. The exemplary axial distributions of the azimuthal Reynolds stress tensor component $\sqrt{\overline{v^{\prime} v^{\prime}}}$ obtained in the middle section of cavities $(L=5, R m=1.8,3.0,5.0, R e=100000)$, Tuliszka-Sznitko et al. [24. 25] see that the turbulence is mostly confined in the stator boundary layer with the maximum at the junction between the stator and the shroud. The turbulent areas are also observed on the rotating inner cylinder, where disturbances coming from the stator are transported towards the rotor. For the higher Reynolds numbers more intense turbulence is also observed in the rotor boundary layer. More information about intensity of the turbulence in particular areas of cavity can be delivered by the distributions of the Reynolds stress tensor components. The exemplary axial distributions of the azimuthal Reynolds stress tensor component $\sqrt{\overline{v^{\prime} v^{\prime}}}$ obtained in the middle section of cavities $(L=5$, $R m=1.8,3.0,5.0, R e=100000)$ are presented in Fig. 4 (prim denotes disturbance and values averaged in time are indicated by overbars). In general [24, 25] all normal Reynolds stress tensor components reach maximum inside the boundary layer. Along with the increasing $R e$ this maximum is located closer to the disk [24, 25]. Outside the boundary layer the values of three components decrease to a value of one order less.

Figure 5 shows the exemplary iso-lines of the dimensionless temperature $\Theta$ obtained for the cavity ( $R e=100000$, $B=0.1, L=5, R m=3.0)$. We can see that the fluid is pumped along the cold rotor $(\Theta(z=1)=0.0)$ towards the heated outer cylinder $(\Theta(r=1)=1.0)$. Then the fluid is transported down and recirculates along the heated stator $(\Theta(z=-1)=1.0)$ towards the inner cylinder. Finally, wormed by stator, the fluid is lifted up along the cold inner cylinder $(\Theta(r=-1)=0.0)$.

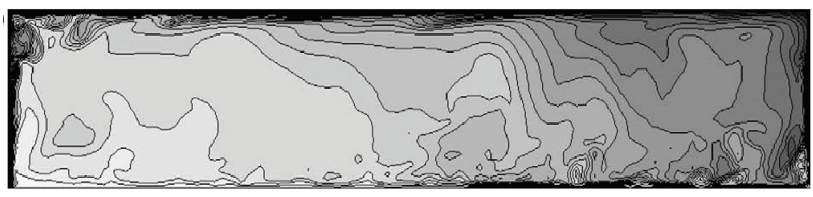

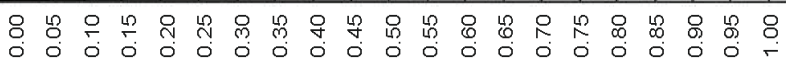

Fig. 5. The isolines of the dimensionless temperature $\Theta$ obtained for the cavity $(R e=100000, B=0.1, L=5, R m=3.0)$. Tuliszka-Sznitko et al. [24]

One of the most important information, from the engineering point of view, is the distribution of the local Nusselt numbers in terms of the radius of the disk. Most of the authors correlate the local Nusselt number with the Reynolds number by a power law such as [14]:

$$
N u_{r}=\frac{\alpha r}{\lambda}=a R e_{r}^{b},
$$


where $\alpha$ is the coefficient of the heat transfer and $\lambda$ is the coefficient of the thermal conductivity, $R e_{r}$ is the local Reynolds number $\operatorname{Re}_{r}=R^{2} \Omega_{1} / v, a$ and $b$ are constants. The coefficient a in the equation (13) depends on the temperature boundary conditions and other characteristic parameters, whereas $b$ changes with the nature of the flow. For laminar flow the Nusselt number varies with radius linearly $(b=0.5)$. In most correlations for turbulent flow authors use $b=0.8$. Exemplary distributions of the local Nusselt numbers in terms of dimensional radius $\bar{r}$ obtained for the different Reynolds numbers are presented in Fig. $6(L=5, R m=3$, $B=0.1)$, [24]. The distribution of the local Nusselt number reflects very well the flow structure: the local Nusselt number reaches maximum at the junction between the stator and the outer cylinder where we observe concentration of turbulence kinetic energy.

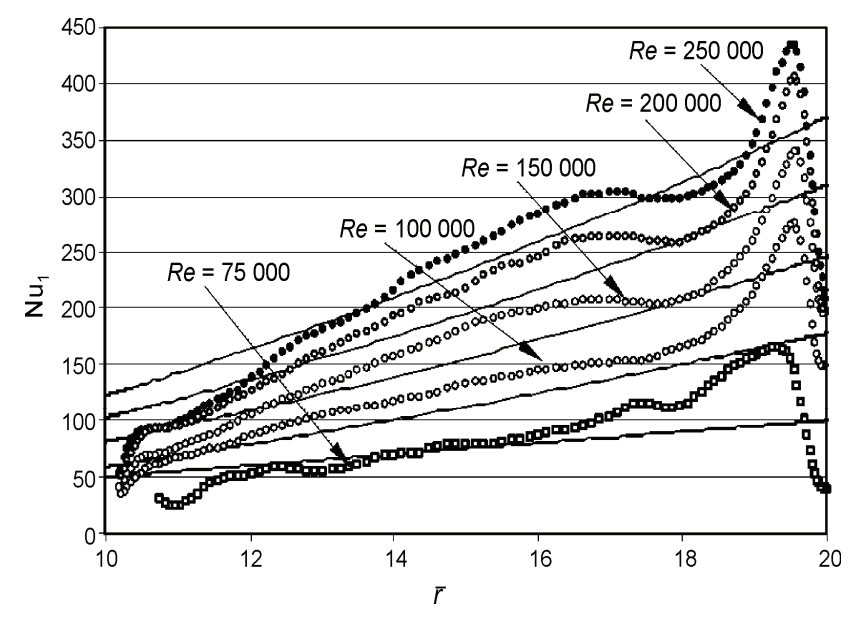

Fig. 6. The local Nusselt number distributions along the stator of cavities $(L=5, R m=3, B=0.1, R e=75000-250000)$. Tuliszka-Sznitko et al. [24]

Correlation coefficients and structural parameters are the most desired for modeling purposes because these parameters are less sensitive to geometrical parameters than the turbulent stresses and heat fluxes. The turbulent Prandtl number $P r_{t}$ and structural coefficients analyzed in paper by Tuliszka-Sznitko et al. [36] are defined as follows:

$$
\begin{gathered}
\operatorname{Pr}_{t}=\frac{\overline{-w^{\prime} v^{\prime}} / \partial v / \partial z}{\overline{w^{\prime} \Theta^{\prime}} / \partial \Theta / \partial z} \\
\left.\overline{\left(v^{\prime 2}\right.}+\overline{u^{\prime 2}}\right) / \overline{w^{\prime 2}} \\
R_{v \Theta}=\left(\overline{v^{\prime} \Theta^{\prime}}+\overline{u^{\prime} \Theta^{\prime}}\right)^{0.5} /\left(\overline{v^{\prime 2}}+\overline{u^{\prime 2}}\right)^{0.5}\left(\overline{\Theta^{\prime 2}}\right)^{0.5}
\end{gathered}
$$

$$
\left.R_{w \Theta}=\overline{w^{\prime} \Theta^{\prime}} / \overline{\left(w^{\prime 2}\right.}\right)^{0.5}\left(\overline{\Theta^{\prime 2}}\right)^{0.5}
$$

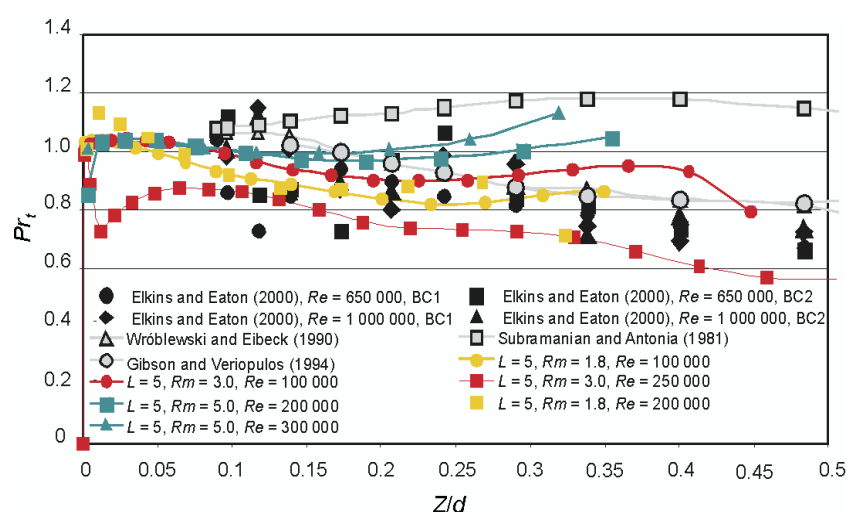

Fig. 7. The turbulent Prandtl $P r_{t}$ number in terms of $Z / \delta$ obtained in the middle section of different configurations (stator boundary layer). The comparison [36] to experimental results taken from the literature $[5,37,38,39]$

Exemplary distributions of the turbulent Prandtl number versus $Z / \delta$ ( $\delta$ denotes the width of the boundary layer) obtained by Tuliszka-Sznitko et al. [36] for the stator boundary layer, and the comparison with experimental results obtained by Elkins and Eaton [5] for a single heated rotating disk, are presented in Fig. 7. We obtained the maximum of $\operatorname{Pr}_{t}$ near the stator $(\sim 1)$ then this value decreased monotonically. Comparison with experimental data obtained by Elkins and Eaton [5] showed good agreement. Elkins and Eaton [5] denoted by BC2 and BC1 (Fig. 7) different thermal conditions).

\section{ROTOR/ROTOR CASE}

In this section we consider the instability structures of the flow between two counter-rotating disks and two cylinders. In the counter-rotating configuration the centrifugal flow induced by the faster rotating disk (upper one) recirculates along the slower rotating disk towards the inner cylinder. This inward recirculation flow meets the outward radial flow, induced by the slower rotating disk, leading to a stagnation area where the radial component of the velocity vanishes. The stagnation area is visible in Fig. 7a and $b$ where in the meridian section the fluid flow and the iso-lines of axial velocity component disturbances obtained for the Reynolds numbers $R e_{2}=3000$ and $R e_{1}=12000$ are shown respectively. With increasing $R e_{1}$ the stagnation area moves from the outer cylinder towards the inner one 
(Tuliszka-Sznitko et al. [40]). For a sufficiently large negative value of $s$, the free shear layer which separates two regions of opposite angular rotations breaks the azimuthal symmetry of the flow. The free shear layer is unstable for the Reynolds number $R e_{1}$ far lower than the critical Reynolds numbers of type II and type I instabilities in both disks boundary layers. The instability of the free shear layer is manifested by the disturbances discovered in experimental work by Gauthier et al. [41] called "negative spirals" (Fig. 8a). The negative spirals slowly rotate with an angular velocity of order of one tenth of the faster rotating disk [40]. The negative spiral vortices obtained in the paper [40] for $R e_{1}=12000, R e_{2}=3000 \quad$ ( $L=9$, $R m=1.5, z=0.84$ ) are presented in Fig. 8b.

a)

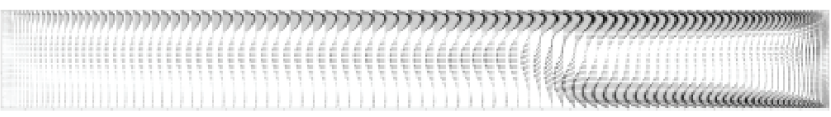

b)



Fig. 7. The flow field (a) and the corresponding iso-lines of axial velocity component disturbances (b) in meridian section obtained for $\left(R e_{1}=12000, R e_{2}=3000, L=9, R m=1.5, z=0.84\right)$. Tuliszka-Sznitko et al. [40]

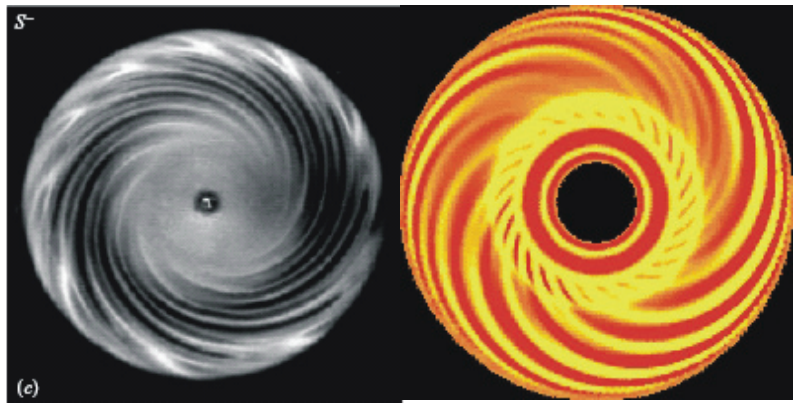

Fig. 8. (a) The experimental results of Gauthier et al. [41], negative spiral vortices, $L=20.9, R m=1$. (b) The iso-lines of azimuthal velocity component disturbances in azimuthal section, $R e_{1}=60000, R e_{2}=3000, z=0.844$, negative spiral vortices.

Tuliszka-Sznitko et al. [40]

\section{RADIAL AND AXIAL OUT-FLOW}

In this section we analyze the flow in the rotor/rotor cavity of the rotational rate $s=1$ with throughflow (both disks rotate in the same direction with the same rotational speed). We consider a radial and an axial outflow of fluid from a source near the inner cylinder to a sink at the outer cylinder. From the turbomachinery air cooling devices point of view examples with the heat transfer are the most interesting. In Fig. 9a and $b$ the exemplary iso-lines of temperature and disturbances of the axial velocity component obtained for the radial outflow $(L=9, R m=3$, $\left.R e_{1}=R e_{2}=200000, B=0.1, C w=800\right)$ are presented respectively. Both disks rotate in the same direction with the same angular speed $(s=1)$. The dimensionless temperature of both disks and of the outer cylinder equals one $\Theta=1$. Temperature of the inner cylinder equals $\Theta=0$. We consider uniform inlet and outlet. Figure 9 shows the effectiveness of radial cooling: the area dominated by the coming cold fluid is laminar.

a)



Fig. 9. (a) The iso-lines of the temperature, (b) the iso-lines of disturbances of the axial velocity component. Radial outflow. $L=9, R m=3, R e_{1}=R e_{2}=200000, B=0.1, C w=800$.

Tuliszka-Sznitko et al. [40]

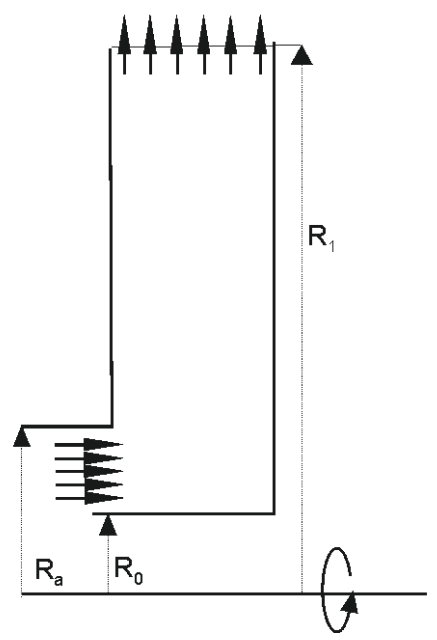

b)

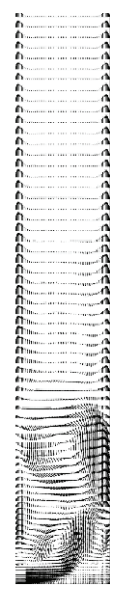

Fig. 10. (a) The schematic picture of the rotating cavity with axial out-flow, b) the flow in meridian section near the source area obtained for $L=9, R m=3, R e_{1}=R e_{2}=200000, B=0$, $a_{2}=0.01594$. Tuliszka-Sznitko et al. [40]

A schematic picture of the cavity with axial inlet is presented in Fig. 10a: air enters the cavity through the write 
hand side disk near the inner cylinder and is extracted through a perforated shroud. We assume uniform inlet and outlet. The flow consists of four regions: the source area, the Ekman layers on both disks, the sink area near the outer cylinder and the inviscid core. Figure10b shows the flow in meridian section the source area obtained for $a_{2}=\left(R_{a}-R_{0}\right) /\left(R_{1}-R_{0}\right)=0.01594(B=0, L=9, R m=3$, $C w=500)$. In the source area intensive redistribution of the flow takes place. Fluid enters the cavity through left disk and in the axial jet impinges on the right disk. Then, the fluid is pumped radialy outward as a wall jet and finally is disgorged to flow radially inward. In the source area two large vortices, which intensify redistribution of the fluid, are observed. Computations have showed that flow in the source is extremely unstationary, which results from oscillations of the axial jet. The obtained meridian flow is in a good agreement with schematic pictures prepared by Owen and Rogers [9, 10] based on their experimental results.

\section{CONCLUSIONS}

This paper contains of the selected results of our numerical investigations on the flow with heat transfer in the rotating cavity. Computations have been performed for different geometrical parameters $L, R m$ and the different Reynolds numbers and thermal conditions. In our computations we used DNS and for the higher Reynolds number LES. In Large Eddy Simulation we used a version of the dynamic Smagorinsky eddy viscosity model proposed by Meneveau et al. [31]. In this version the value of $C_{S}$ is averaged for some time along the fluid particle pathlines. This algorithm turned out to be very effective and allowed us to perform computations for higher Reynolds numbers. We analyzed three configurations: rotor/stator, counterrotating rotor/rotor and co-rotating rotor/rotor with throughflow.

In rotor/stator configurations the investigated flows belong to the Batchelor family, i.e. the flows are divided into two boundary layers separated by a central rotating inviscid core. For rotor/stator configuration fluid turbulence concentrates in the stator boundary layer and its intensity increases towards the outer cylinder. The instability structures and the level of turbulence depend on the Reynilds number and on the geometrical parameters: curvature parameter and aspect ratio. We focused on the analysis of the distributions of the Reynolds stress tensor components, the correlation coefficients and structural parameters. The maximum values of the turbulent Prandtl number $\left(\operatorname{Pr}_{t}=0.8-1.2\right)$ we obtained near the stator [36].

In counter-rotating configurations we focused on instability structure. Detailed analysis of different instability structures which appear in this type of flow can be found in paper [40]. Three different instability patterns have been found: axisymmetric propagating vortices interpreted as type II instability, positive spiral vortices interpreted as type I instability and negative spirals. The negative spirals rotate around the central axis and the mechanism is not of the cross-flow type; it is probably the result of an interaction between free shear layer and the Ekman layer. These computations we discussed in the light of the experimental results of Gauthier et al. [41].

The most interesting, from application point of view, are configurations with the radial outflow (flow with the source and sink). In the frame of radial out-flow computations have been performed for radial and axial inlet [40]. Meridian flow showed good agreement with Owen and Rogers [9, 10] experimental results. Computations performed for the co-rotating disks with superimposed cooling air showed effectiveness of the radial cooling.

\section{References}

[1] J.W. Daily, R.E. Nece, Chamber dimension effects on induced flow and friction resistance of enclosed rotating disks. J. Basic Engng. 82, 217-232 (1960).

[2] S.T. McComas, J.P. Hartnett, Temperature profiles and heat transfer associated with a single disk rotating in still air. Proc. 4th Heat Transfer Conf., Versailles, 3, FC7.7 (1970).

[3] C.O. Popiel, L. Boguslawski, Local heat-transfer coefficients on the rotating disk in still air. Int. J. Heat Mass Transfer 18, 167-170 (1975).

[4] H.S. Littell, J.J. Eaton, Turbulence characteristics of the boundary layer on a rotating disk. J. Fluid Mech. 266, 175-207 (1994).

[5] C.J. Elkins, J.K. Eaton, Turbulent heat and momentum transport on a rotating disk. J. Fluid Mech. 402, 225-253 (2000).

[6] N.I. Nikitenko, Experimental investigation of heat exchange of a disk and screen. J. Eng. Phys. 6, 1-11 (1963).

[7] J.M. Owen, J.R. Pincombe, Velocity measurements inside a rotating cylindrical cavity with a radial outflow of fluid. J. Fluid Mech. 99, 111-127 (1980).

[8] V.K. Schukin, V.V. Olimpiev, Heat transfer of disc rotating in a housing with transitional and turbulent boundary layer. Soviet Aeronaut. 18, 77-81 (1975).

[9] J. M. Owen, R. Rogers, Flow and heat transfer in rotatingdisc systems. vol. 1. Research Studies Press Taunton (1989).

[10] J.M. Owen, R.H. Rogers, Flow and heat transfer in rotating-disc systems. vol. 2. Research Studies Press Taunton (1995). 
[11] S. Mochizuki, W. Yang, Three mechanisms of convective enhancement in stationary disk systems. Trans. ASME J. Heat Transfer 108, 978-980 (1986).

[12] M. Itoh, Y. Yamada, S. Imao, M. Gonda, Experiments on turbulent flow due to an enclosed rotating disk. In: Ganic, E., Rodi, W. (Eds.), Engineering Turbulence Modeling and Experiments, Elsevier, New York, 659-668 (1990).

[13] M. Djaoui, R. Debuchy, Heat transfer in a rotor-stator system with a radial inflow. C.R. Acad. Sci. Paris II B. 326, 309-314 (1998).

[14] J. Pellé, S. Harmand, Heat transfer measurements in an opened rotor-stator system air-gap. Exp. Therm. Fluid Sci. 31, 165-180 (2007).

[15] A. Morse, Numerical prediction of turbulent flow in rotating cavities. In: Gas Turbine Conf. and Exhibition, Anaheim, CA, ASME Paper 87-GT-74 (1987).

[16] B.E. Launder, B.I. Sharma, Application of the energy dissipation model of turbulence to the calculation of flow near a spinning disc. Letters in Heat and Mass Transfer. 1(2), 131-138 (1974).

[17] J.W. Chew, C.M. Vaughan, Numerical predictions for the flow induced by an enclosed rotating disc. In: 33rd Gas Turbine Conf., Amsterdam, ASME Paper 88-GT-127, (1988).

[18] E. Serre, J.P. Pulicani, A three-dimensional pseudospectral method for rotating flows in a cylinder. Comput. Fluids 30, 491 (2001).

[19] E. Serre, E. Tuliszka-Sznitko, P. Bontoux, Coupled numerical and theoretical study of the transition flow between a rotating and stationary disk, Phys. Fluids 16 (3), 688-707, (2004).

[20] F. Moisy, O. Doaré, T. Pasutto, O. Daube, M. Rabaud, Experimental and numerical study of the shear layer instability between two counter-rotating disks. J. Fluid Mech. 507, 175-202 (2004).

[21] R. Jacques, P. Le Quéré, O. Daube, Axisymmetric numerical simulations of turbulent flow in a rotor stator enclosures. Int. J. Heat Fluid Flow. 23, 381-397 (2002).

[22] A. Randriamampianina, S. Poncet, Turbulence characteristics of Bödewadt layer in a large enclosed rotor-stator system. Phys. Fluids 18, 055104 (2006).

[23] E. Tuliszka-Sznito, E. Serre, P. Bontoux, On the nature of the boundary layers instabilities in a flow between a rotating and a stationary disc. C.R. Acad. Sci. Paris 330, 91-99 (2002).

[24] E. Tuliszka-Sznitko, A. Zieliński, W. Majchrowski, LES and DNS of the non-isothermal transitional flow in rotating cavity. Int. J. Heat and Fluid Flow. 30 (3), 534-548 (2009).

[25] E. Tuliszka-Sznitko, A. Zieliński, W. Majchrowski, LES of the transitional flow in rotor/stator cavity. Archive of Mechanics 61(2), 93-118 (2009).
[26] M. Lygren, H.I. Andersson, Turbulent flow between a rotating and a stationary disk. J. Fluid Mech. 426, 297 (2001).

[27] X. Wu, K.D. Squires, Prediction and investigation of the turbulent flow over a rotating disk. J. Fluid Mech. 418, 231-264 (2000).

[28] H.I. Andersson, M. Lygren, LES of open rotor-stator flow. Int. J. Heat Fluid Flow 27 (4), 551-557 (2006).

[29] E. Séverac, S. Poncet, E. Serre, M.P. Chauve, Large eddy simulations and measurements of turbulent enclosed rotorstator flows. Phys. Fluids 19, 085113-1-17 (2007).

[30] A. Randriamampianina, P. Bontoux, B. Roux, Ecoulements induits par la force gravifique dans une cavité cylindrique en rotation. Int. J. Heat Mass Transfer 30 (7), 1275-1292 (1987).

[31] C. Meneveau, T.S. Lund, W.H. Cabot, A Lagrangian dynamic subgrid-scale model of turbulence. J. Fluid Mech. 319, 353-385 (1996).

[32] E. Serre, E. Crespo del Arco, P. Bontoux, Annular and spiral patterns between a rotating and a stationary disk. J. Fluid Mech. 434, 65-100 (2000).

[33] M. Germano, U. Piomelli, P. Moin, W. Cabot, A dynamic subgrid-scale eddy-viscosity model. Phys. Fluids A, 3 (7), 1760-1765 (1991).

[34] D.K. Lilly, A proposed modification of the Germano subgrid-scale closure method. Phys. Fluids A, 4 (3), 633-635 (1992).

[35] E. Tuliszka-Sznitko, W. Majchrowski, Large eddy simulation of non-isothermal flow in rotor/stator cavity. Proceedings of Int. Symp. on Heat Transfer in Gas Turbine Systems (2009).

[36] E. Tuliszka-Sznitko, A. Zielinski, W. Majchrowski, LES of the non-isothermal flow in rotating cavity. Springer, Hybrid RANS-LES Methods, ed. by W. Haase (accepted) (2009).

[37] D.E. Wróblewski, P.A. Eibeck, An experimental investigation of turbulent heat transport in a boundary layer with an embedded streamwise vortex. $\mathrm{PhD}$ Thesis, University of California at Berkeley, Mechanical Engineering Department (1990).

[38] M.M. Gibson, C.A. Verriopoulos, Turbulent boundary layer on a middle curved surface. Part 2: Temperature field measurements, Exps. Fluids 2, 73-80 (1984).

[39] C.S. Subramanian, R.A. Antonia, Effect of Reynolds number on a slightly heated turbulent boundary layer. Int. J. Heat Mass Transfer 24, 1833-1846 (1981).

[40] E. Tuliszka-Sznitko, A. Zielinski, DNS/LES of Transitional Flow in Rotating Cavity. Int. J. of Transport Phenomena 10(3), 223-234 (2008).

[41] G. Gauthier, P. Gondret, F. Moisy, M. Rabaud, Instabilities in the flow between co- and counter-rotating disks. J. Fluid Mech. 473, 1-21 (2002). 


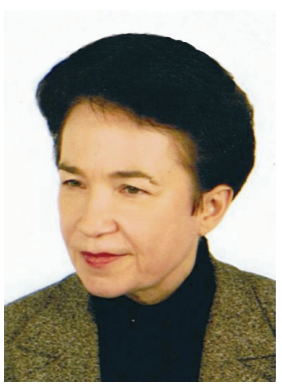

Ewa Tuliszka-Sznitko, Professor of Poznan University of Technology (Ph.D. 1988, Dr. Habil. 1993, Institute of Fluid flow Machineries Polish Academy of Sciences). Member of European Community of Flow Turbulence and Combustion. Research interests: computational fluid dynamics, instability, turbulence. Authors of about 100 papers published among others in Physics of Fluids, Heat and Fluid Flow, International Journal of Transport Phenomena, CRASP. Lectures on Thermodynamics, Fluid dynamics, Management of Energy. Invited professor at Les Universites d' Aix-Marseille.



WoJCiech MaJChrowSKI, MSc. was born 21.07.1982 in Słupsk, Poland. Graduated from Mathematics at Poznan University of Technology. Actually a PhD student at Poznan University of Technology in Faculty of Working Machines and Transportation. The main field of interests: numerical simulation of fluid flow in rotating cavities using both DNS and LES methods. 